\title{
Article
}

\section{The edible wide mushrooms of Agaricus section Bivelares from Western China}

\author{
Zhang MZ ${ }^{1,2}$, Li GJ ${ }^{1}$, Dai RC ${ }^{1}$, Xi YL ${ }^{3}$, Wei SL ${ }^{3 *}$ and Zhao $\mathrm{RL}^{1,2^{*}}$ \\ ${ }^{1}$ State Key Laboratory of Mycology, Institute of Microbiology, Chinese Academy of Sciences, No3 1st Beichen West \\ Road, Chaoyang District, Beijing 100101, China \\ ${ }^{2}$ College of Life Sciences, University of Chinese Academy of Sciences, Huairou District, Beijing 101408, China \\ ${ }^{3}$ Gansu Engineering Laboratory of Applied Mycology, Hexi University, 846th Huancheng North Road, Ganzhou \\ District, Zhangye 734000, Gansu, China
}

Zhang MZ, Li GJ, Dai RC, Xi YL, Wei SL, Zhao RL 2017 - The edible wide mushrooms of Agaricus section Bivelares from Western China. Mycosphere 8(10), 1640-1652, Doi $10.5943 /$ mycosphere/8/10/4

\begin{abstract}
Agaricus is a genus of macrofungi containing species with highly edible and medicinal values. A mushroom survey was recently carried out in Qilian Mountain National Natural Reserve, in Gansu Province of China, and yielded 21 Agaricus specimens. The morphological examination and phylogenetic analysis based on four-gene sequences from those specimens were conducted. The result shows they belong to four species in A. section Bivelares: A. sinotetrasporus sp. nov. and A. qilianensis sp. nov. are new species for science; $A$. devoniensis is a new record from China; and the famous button mushroom, A. bisporus is found in the wild. All of them are described and illustrated in details. A brief comparison with similar taxa or previous records are addressed too.
\end{abstract}

Key words - Agaricaceae - Multi-gene - Phylogeny - Taxonomy

\section{Introduction}

Agaricus L. is the type genus of the family Agaricaceae in the phylum Basidiomycota, it presently includes more than 500 edible or poisonous species (Zhao et al. 2016). The genus is distributed worldwide, and several species are served as food, including the commercially cultivated species such as $A$. bisporus (J.E. Lange) Imbach, the famous button mushroom, and $A$. subrufescens Peck, the almond mushroom (syn. A. blazei Murrill sensu Heinemann) and many non-cultivated species, such as A. campestris L. and A. augustus Fr. In recent years, several species, which have been described as new for science are consumed by local people for a long time or have potential edible and medicinal values, such as A. flocculosipes R.L. Zhao, Desjardin, Guinb. and K.D. Hyde (Zhao et al. 2012), A. sinodeliciosus Z.R. Wang and R.L. Zhao (Wang et al. 2015), and A. taeniatus Sai F. Li, Shao J. Li and H.A. Wen (Li et al. 2014).

Agaricus species have considerable ecological, nutritional and medicinal interests, yet the extent of its diversity remains poorly know in some areas, particularly in subtropical and tropical areas. The classification of Agaricus species was previously mainly based on species from the temperate regions of the north hemisphere and comprised eight widely accepted sections (Cappelli 1984, Parra 2008, 2013). After the inclusion of tropical samples, eleven potentially new sections were revealed by phylogenetic analysis based on ITS sequence data (Zhao et al. 2011). The recently 
reconstructed taxonomic system of Agaricus based on multi-gene sequences and molecular dating analysis, recognizes this genus as five subgenera and twenty sections (Zhao et al. 2016). Then Chen et al. (2017) added a sixth subgenus in this classification.

Even the taxonomic system of Agaricus has been updated largely in recent years, $A$. section Bivelares is keeping stable with its well morphological and molecular definitions. Section Bivelares was first described by Kauffman under the name of "Psalliota", and typified by P. rodmanii (Peck) Kauffman [Syn. of A. bitorquis (Quél.) Sacc.]. In 1897, Karsten formally transfer this genus under the older name of Agaricus L. Fr. ss. Karsten (=Psalliota Fr.) (Cappelli 1984), so section Bivelares moved into the genus Agaricus. The later studies well reorganized this section using morphological characters (Heinemann 1978, Wasser 1980, Cappelli 1984, Kerrigan 1986). Recent years, with the development of molecular biology, molecular phylogenetic analyses results support this section well (Challen et al. 2003, Didukh et al. 2004, Kerrigan 2008) and it was slightly modified as A. sect. Bivelares (Kauffman) L.A. Parra and characterized by negative Schäffer and KOH reactions, red discoloration of the context, a mild or indistinct odor and edibility (Parra 2008). In the new system of classification recently proposed, A. sect. Bivelares is one of the 12 sections of the $A$. subg. Pseudochitonia (Zhao et al. 2016).

Qilian Mountains National Natural Reserve locates at western China with the east longitude $97^{\circ} 25^{\prime} \sim 103^{\circ} 38^{\prime}$, North latitude $36^{\circ} 45^{\prime} \sim 39^{\circ} 40^{\prime}$, at an altitude of $2000 \mathrm{~m}-4500 \mathrm{~m}$, with an alpine arid and semi-arid climate (Xi 2011). The sampling area was in the central area of the eastern slope of the Qilian Mountains, belonging to the continental cold and semi-arid and semi-humid forest grassland climate. We made a mushrooms survey on this area in 2016, and the sampling sites are about 2800-3000 meters above sea level. At this altitude, Picea crassifolia is the preponderant tree in those primary forests and scatter some shrub at the edge areas of the forests.

\section{Materials \& Methods}

\section{Morphological characters examination}

Specimens were collected and photographed in situ and then kept separately in a box or packed by aluminum foil. Macro-morphological characters and Macro-chemical reactions were recorded on fresh specimens. Specimens were dried overnight in a food drier and sealed in plastic bags, then brought back laboratory. The micro-morphological observation was carried out under microscopy. Attention was paid to the basidiospores, basidia and cystidia and the anatomy of the pileipellis. Measurements of basidiospores, basidia and cheilocystidia are presented based on at least 20 measurements, and include the range of spore length by width $\mathrm{x}$, the mean of all spores \pm SD (Standard Deviation); Q, the range of the quotient length/width of all basidiospores and Qm, the mean $\mathrm{Q}$ of all spores \pm SD. Specimens cited were deposited in Herbarium Mycologicum Academiae Sinicae (HMAS), Institute of Microbiology, Chinese Academy of Sciences, Beijing, China.

\section{Molecular phylogenetic analysis}

DNA extractions were made with a DNA extraction kit (Broad spectrum plant genomic DNA rapid extraction kit, Biomed, China). ITS1-5.8S-ITS2 region (ITS) of rDNA region were amplified by PCR reactions with primers ITS1/ITS4 or ITS5/ITS4 (White et al. 1990, Gardes \& Bruns 1993), Large Subunit of rDNA region (LSU) amplified with primers LROR/LR5, elongation factor-1 $\alpha$ (EF1) gene sequence amplified with primers 983F/1953R, mitochondrial intermediate peptidase (MIP) gene sequence amplified with primers 91BF/91BR based on the previous studies (Thongklang et al. 2014, Zhao et al. 2016). All PCR products were sent to commercial biotechnological company (Biomed Co. Ltd, Beijing) for sequencing. The alignment is deposited to TreeBase (submission ID: 21021). (Reviewer access URL: http://purl.org/phylo/treebase/phylows/study/TB2:S21021?x-access code $=9 f 75 b 34777 b 3 e f 3 c 637$ feed580be30d7\&format $=$ html) 
The datasets were constructed by sequences from authority specimens retrieved from GenBank, along with those sequences produced from this study (indicated in Table 1). Sequences of each gene were aligned using MUSCLE (http://www.ebi.ac.uk/Tools/msa/muscle/) separately, cut off the head and tail fragments to make it consistent in length. Then those four genes sequences were combined by SequenceMatrix1.7.8 (Gaurav 2010). Maximum likelihood (ML) phylogenetic tree and bootstrap values (BS) obtained from 1000 replicates were performed using RAxmlGUI 1.3 (Silvestro \& Michalak 2012). Bayesian analyses were performed using MrBayes v3.2.6 (Ronquist \& Huelsenbeck 2003) with the substitution model selected by MrModeltest 2.3 (Johan A. A. Nylander 2004). Four simultaneous Markov chains were run for 2,000,000 generations and trees were sampled every 100th generation. The first 5,000 resulting trees were discarded as burn-in and the remaining trees were used to calculate Bayesian posterior probabilities (PP) in the majority rule consensus tree. Trees were viewed in FigTree1.4.3 (Andrew Rambaut 2006).

Table 1 Materials used in the phylogenetic analysis.

\begin{tabular}{|c|c|c|c|c|c|c|}
\hline Collection NO. & Species & LSU GenBank & ITS GenBank & EF1 GenBank & $\begin{array}{l}\text { MIP } \\
\text { GenBank }\end{array}$ & Location \\
\hline RWK1397_T & A. agrinferus & - & EU257801 & - & - & California, USA \\
\hline Bs423_T & A. bisporus & - & KF848699 & - & - & $\begin{array}{l}\text { Olonne-sur-mer, } \\
\text { France }\end{array}$ \\
\hline Bs261 & A. bisporus & - & AF465404 & - & - & Dinard, France \\
\hline JB3-83 & A. bisporus & - & AF465401 & - & - & California, USA \\
\hline MYA-4627 & A. bisporus & - & GU327643 & - & - & California, USA \\
\hline ZRL20160021 & A. bisporus & KY885133 & KY885112 & KY905103 & KY905124 & Gansu, China \\
\hline ZRL20161813 & A. bisporus & KY885138 & KY885117 & KY905108 & KY905129 & Gansu, China \\
\hline ZRL20161854 & A. bisporus & KY885139 & KY885118 & KY905109 & KY905130 & Gansu, China \\
\hline ZRL20162049 & A. bisporus & KY885143 & KY885122 & KY905113 & KY905134 & Gansu, China \\
\hline LAPAG446 & A. bisporus & KR006611 & KM657920 & KR006640 & - & Burgos, Spain \\
\hline LAPAG486 & A. bisporus & - & KM657921 & - & - & - \\
\hline WZR2012827 & A. bitorquis & KY885130 & KY885109 & KY905100 & KY905121 & Gansu, China \\
\hline CA427 & A. bitorquis & КT951491 & КT951320 & КT951646 & - & - \\
\hline RWK1462 & A. bitorquis & - & AF432898 & - & - & USA \\
\hline LAPAG889 & A. cupressicola & KT951465 & KT951334 & КT951649 & - & Roma, Italy \\
\hline G84.112_T & A. cupressicola & - & EU363031 & - & - & Italy \\
\hline FS-22_T & $\begin{array}{l}\text { A. } \\
\text { cupressophilus }\end{array}$ & - & EU258676 & - & - & California, USA \\
\hline ZRL20162196 & A. devoniensis & KY885147 & KY885126 & KY905117 & KY905138 & Gansu, China \\
\hline CA445 & A. devoniensis & - & EU363036 & - & - & Sardegna, Italy \\
\hline ZRL20161021_T & A. qilianensis & KY885135 & KY885114 & KY905105 & KY905126 & Gansu, China \\
\hline ZRL20161024 & A. qilianensis & KY885136 & KY885115 & KY905106 & KY905127 & Gansu, China \\
\hline ZRL20161797 & A. qilianensis & KY885137 & KY885116 & KY905107 & KY905128 & Gansu, China \\
\hline ZRL20161019 & A. qilianensis & - & - & - & - & Gansu, China \\
\hline ZRL20161810 & A. qilianensis & - & - & - & - & Gansu, China \\
\hline ZRL20162094 & A. qilianensis & - & - & - & - & Gansu, China \\
\hline WZR2012821_T & A. sinodeliciosus & KY885129 & KY885108 & KY905099 & KY905120 & Gansu, China \\
\hline ZRL20152598 & A. sinodeliciosus & KY885131 & KY885110 & KY905101 & KY905122 & Gansu, China \\
\hline ZRL20160001 & A. sinodeliciosus & KY885132 & KY885111 & KY905102 & KY905123 & Gansu, China \\
\hline ZRL20161020_T & $\begin{array}{l}\text { A. } \\
\text { sinotetrasporus }\end{array}$ & KY885134 & KY885113 & KY905104 & KY905125 & Gansu, China \\
\hline ZRL20161862 & $\begin{array}{l}\text { A. } \\
\text { sinotetrasporus }\end{array}$ & KY885140 & KY885119 & KY905110 & KY905131 & Gansu, China \\
\hline ZRL20162148 & $\begin{array}{l}\text { A. } \\
\text { sinotetrasporus }\end{array}$ & KY885144 & KY885123 & KY905114 & KY905135 & Gansu, China \\
\hline
\end{tabular}


Table 1 Continued.

\begin{tabular}{lllllll}
\hline Collection NO. & Species & LSU GenBank & ITS GenBank & EF1 GenBank & $\begin{array}{l}\text { MIP } \\
\text { GenBank }\end{array}$ & Location \\
\hline ZRL20162192 & $\begin{array}{l}\text { A. } \\
\text { sinotetrasporus }\end{array}$ & KY885146 & KY885125 & KY905116 & KY905137 & Gansu, China \\
ZRL20162019 & A. $\boldsymbol{s p}$ & KY885141 & KY885120 & KY905111 & KY905132 & Gansu, China \\
ZRL20162178 & A. $\boldsymbol{s p}$ & KY885145 & KY885124 & KY905115 & KY905136 & Gansu, China \\
CA103 & A. sp & - & EU363037 & - & - & Oléron \\
RWK1994 & A. subfloccosus & - & EU131640 & - & France \\
RWK1701 & A. subperonatus & - & AF432902 & - & - & New Mexico, USA \\
RWK1789 & A. subsubensis & - & EU257802 & - & - & Toronto, Canada \\
QLS60_T & A. taeniatus & KY885127 & KJ623317 & KY905097 & KY905118 & Gansu, China \\
QLS15 & A. taeniatus & KY885128 & KJ623319 & KY905098 & KY905119 & Gansu, China \\
CA221_T & A. tlaxcalensis & - & EU363033 & - & - & Tlaxcala, Mexico \\
CA339_OUT & A. gennadii & - & KT951318 & KT951575 & - & 8) \\
LAPAG257_OU & A. nevoi & KR006606 & KM657922 & KR006635 & - & Burgos, Spain \\
T & & & B & Burgos, Spain \\
\hline
\end{tabular}

T refers to holotype; OUT refers outgroup; Bold refers to the sequences produced from this study.

\section{Results}

The dataset consists of 4 genes sequences from 43 specimens representing 15 species of $A$. section Bivelares and two species of the close related section A. sect. Chitonioides, A. gennadii and A. nevoi, which were chosen as outgroups for rooting purposes (details see Table 1). The dataset consists of 2346 characters, of which 2055 are constant, 76 variable characters are parsimonyuninformative and 215 are parsimony-informative. The ML and Bayesian topologies were almost identical and the Bayesian tree was showed in Fig. 1. All taxa of A. section Bivelares form a wellsupported monophyletic lineage (100\% BS, $100 \% \mathrm{PP})$, and sister to the outgroup taxa A. gennadii and $A$. nevoi.

Specimen ZRL20162196 nests with CA445 (A. devoniensis) under a fully supports (100\% BS, 1 PP). Specimens ZRL20161020, ZRL20161862, ZRL20162148 and ZRL20162192 form a monophyletic clade under the statistic supports of $97 \%$ BS, 1 PP, which represents as a new species A. sinotetrasporus in this study. Specimens ZRL20161019, ZRL20161021, ZRL20161024, ZRL20161797 and ZRL20161810, ZRL20162094 form another monophyletic clade under the statistic supports of $80 \% \mathrm{BS}, 0.99 \mathrm{PP}$, which represents as another new species A. qilianensis. Specimens ZRL20160021, ZRL20161813, ZRL20161854 and ZRL20162049 cluster with A. bisporus from Europe and North America under 99\% BS, 1 PP supports.

\section{Taxonomy}

Agaricus sinotetrasporus Y.L. Xi, M.Z. Zhang \& R.L. Zhao, sp. nov.

Fig. 2

Fungal Names: FN570462

Etymology - the epithet of "sino" refers to the location of this species from China and "tetrasporus" refers to the 4-spored basidia.

Typus - China, Gansu Province, Zhangye City, Qilian Mountain National Natural Reserve, collected by R.L. Zhao, 31 August 2016, ZRL20161020 (HMAS 255155, holotype).

Pileus $20-40 \mathrm{~mm}$ in diam. parabolic, pulvinate, top flat when young; then $85-95 \mathrm{~mm}$ in diam. when mature, convex, plano-convex, centre subumbonate or slightly depressed, circular margin inrolled to decurved in age, exceeding, crenate in some cases; surface dry, covered by scales completely, appressed, often reddish brown or dark brown, sometimes brown. 


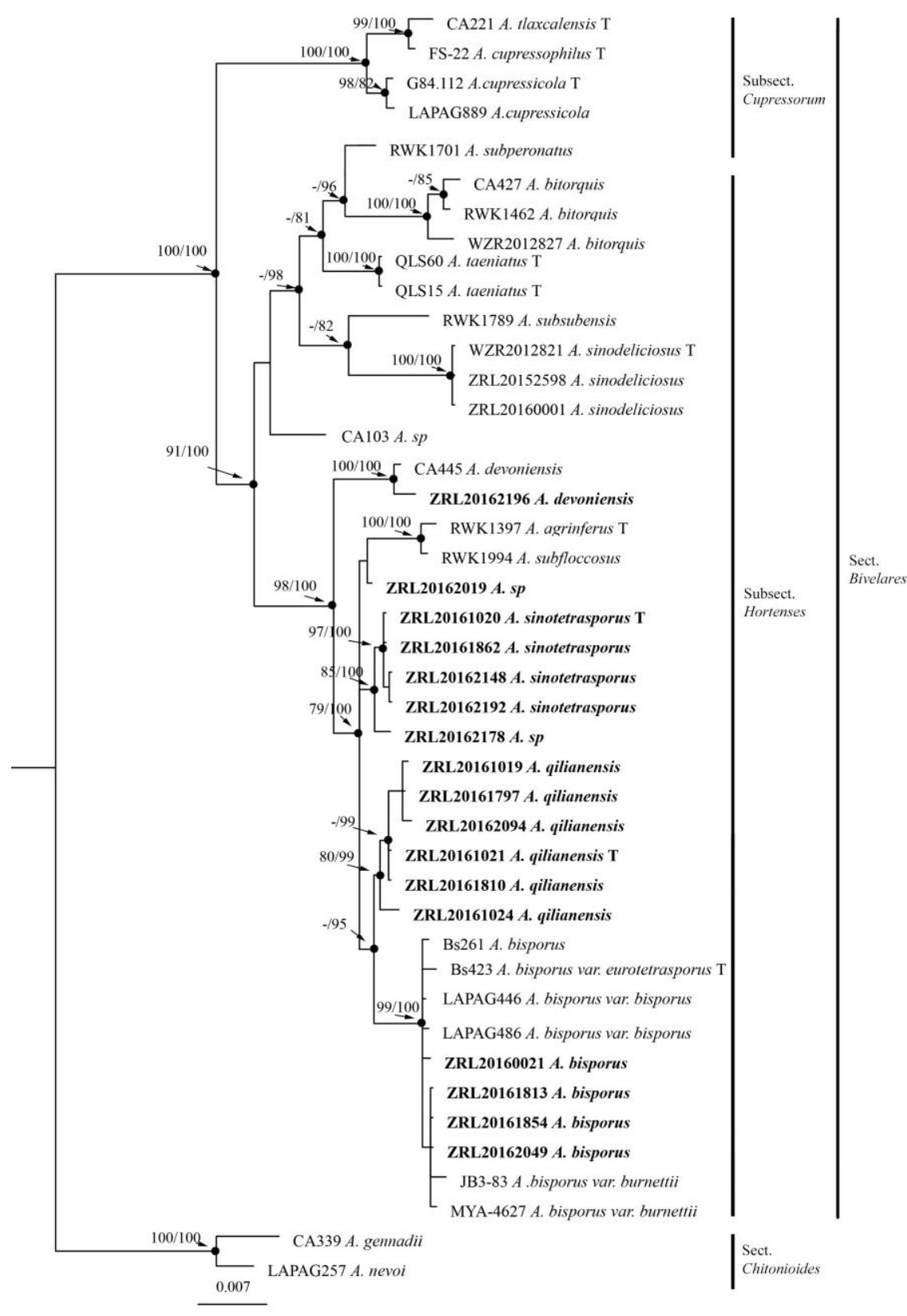

Figure 1 - Phylogeny of Agaricus section Bivelares generated from Bayesian analysis of four genes sequences, A. gennadii (CA339) and A. nevoi (LAPAG257) are outgroups. Parsimony bootstrap (BS) and Bayesian posterior probability (PP) values $>79 \%$ are given at the internodes (BS/PP). Bolt refers to the sequences produced from this study; T refers to the type specimen.

Lamellae free, crowded, unequal, pink, brown to dark brown. Stipe $25-40 \times 15-20 \mathrm{~mm}$ when young 55-65 × 8-11(apex) - 25-30 (base) $\mathrm{mm}$, cylindrical, clavate, solid to narrow hollow; surface fibrillose and heavily fibrillose scales towards base, erected, somehow concentric, white. Annulus membranous, superous, cottonous, thick, double, upper side often striate and lower side cogwheel towards margin, up to $15 \mathrm{~mm}$ broad, pendant, upper side smooth or slightly striate, white, lower side fibrillose and form discrete squames, white or light brown at the edge; Context 6-8 mm thick at the disc, white. Slightly reddish brown on scratching, and distinct rubescent discoloration on cutting. Odour pleasant, mushroomy.

Macrochemical reactions $-\mathrm{KOH}$ and Schäffer's reactions negative.

Basidiospores (5.8-) 6.3-7.6 $\times 4.5-5.7 \mu \mathrm{m}\left[\mathrm{x}=6.9 \pm 0.4 \times 5.0 \pm 0.3, \mathrm{Q}=1.2-1.6, \mathrm{Q}_{\mathrm{m}}=1.4 \pm\right.$ $0.1, \mathrm{n}=20$ ], ellipsoid, brown, smooth and thick-walled, without germ pore. Basidia 24-29 $(-33) \times$ 7.6-12 $\mu \mathrm{m}$, clavate, mostly 4-spored and rarely 2-spored, hyaline. Cheilocystidia $27-35 \times 13-$ 
$16 \mu \mathrm{m}$, mostly clavate, pyriform in some cases with long stipe, hyaline. Pleurocystidia absent. Pileipellis a cutis composed of hyphae 6.7-11 $\mu \mathrm{m}$ in diam., long cylindrical, curved, unbranched, containing brown pigments.

Habitat - scattered in Picea crassifolia forest.

Other materials examined - China, Gansu Province, Zhangye City, Sunan county, Qilian Mountain National Natural Reserve, Kangle, collected by R.L. Zhao, 28 August 2016, ZRL20161862 (HMAS 281180); Gansu Province, Zhangye City, Sunan county, Qilian Mountain National Natural Reserve, Dayekou reserve station, Alt. 2810 2980m, E 100¹4'31.13", N 38 32'52.71", collected by R.L. Zhao, 31 August 2016, ZRL20162148 (HMAS 255153), ZRL20162192 (HMAS 281181).

Notes - This new species is similar to A. bisporus in morphology because both present a variable colour on pileus, such as from reddish brown, dark brown to near white; similar basidiospores and cheilocystidia in shape and size (Parra 2013). Furthermore, the tetrasporic basidia and basidiospores with an average of longer than $6 \mu \mathrm{m}$ make A. sinotetrasporus similar to A. bisporus var. eurotetrasporus mostly (Callac et al. 2003). The distinct morphological characters to separate this new species from the known variety A. bisporus var. eurotetrasporus is the character of annulus, which is double and superous in new species vs single and intermediate or inferous for A. bisporus (Parra 2008). On the other hand A. sinotetrasporus only known from western China, while A. bisporus var. eurotetrasporus only known from Europe (Callac et al. 2003; Parra 2008). In the molecular phylogeny, the topology clearly shows A. sinotetrasporus is not related to A. bisporus var. eurotetrasporus which is represented by its type specimen Bs423 (Fig. 1). Then we propose those Chinese specimens as a novel species and characterized by 4-spored basidia and its Asia distribution. A preliminary cloning experiment for its MIP gene PCR amplification products shows the presence of SNP (Single nucleotide polymorphyism). Then we could exclude that $A$. sinotetrasporus has a homothallic life cycle.

The unnamed specimen ZRL20162178 has a sister position with A. sinotetrasporus clade (Fig. 1) and phylogenetically it was possibly the same species to this new species. However we did not named it, as we lack its morphological information.

Agaricus qilianensis S.L. Wei, M.Z. Zhang \& R.L. Zhao, sp. nov.

Fig. 3

Fungal Names: FN570460

Etymology - epithet "qilian" refers to Qilian Mountain where the type specimen is from.

Typus: China, Gansu Province, Zhangye City, Sunan county, Qilian Mountain National Natural Reserve, Kangle, collected by R.L. Zhao, 28 August 2016, ZRL20161021 (HMAS 255156, holotype).

Pileus 30-45 mm when young, 97-102 $\mathrm{mm}$ in diam. parabolic to cushion when young, then convex, plano-concave, centre slightly depressed in old, margin straight and entire; surface dry, covered by fibrils completely, broken into fine squamoses towards the margin, and often woolly at the margin, brown or light brown against white background. Lamellae free, crowded, unequal, pink, reddish brown to dark brown in age. Stipe 15-25 $\mathrm{mm}$ when young; 65-75 $\times 9-11 \mathrm{~mm}$, equal with a round base, solid, surface, fibrillose squamose, and floccose when young below the ring. Annulus pendant, membranous, thick, median, entire, pendant or subperonate, white, 5-7 $\mathrm{mm}$ in diam; Context 7-10 mm thick at the disc, white. Reddish brown on touching; rubesent on cutting. Odour pleasant, mushroomy.

Macrochemical reactions $-\mathrm{KOH}$ and Schäffer's reactions negative.

Basidiospores 6.0-7.3 × 4.6-6.5 $\mu \mathrm{m}[\mathrm{x}=6.6 \pm 0.4 \times 5.5 \pm 0.4, \mathrm{Q}=1.1-1.4, \mathrm{Qm}=1.2 \pm 0.1, \mathrm{n}$ $=20]$, ellipsoid, brown, smooth and thick-walled, without germ pore. Basidia 22-33 $\times 7.5-12 \mu \mathrm{m}$, clavate, 2-spored, hyaline. Cheilocystidia $17-41 \times 7.9-14 \mu \mathrm{m}$, mostly clavate, pyriform in some cases with narrow stipe, hyaline. Pleurocystidia absent. Pileipellis a cutis composed of hyphae 4.5$15.9 \mu \mathrm{m}$ in diam., long cylindrical, curved, branched, containing brown pigments.

Habitat - scattered in Picea crassifolia forest. 

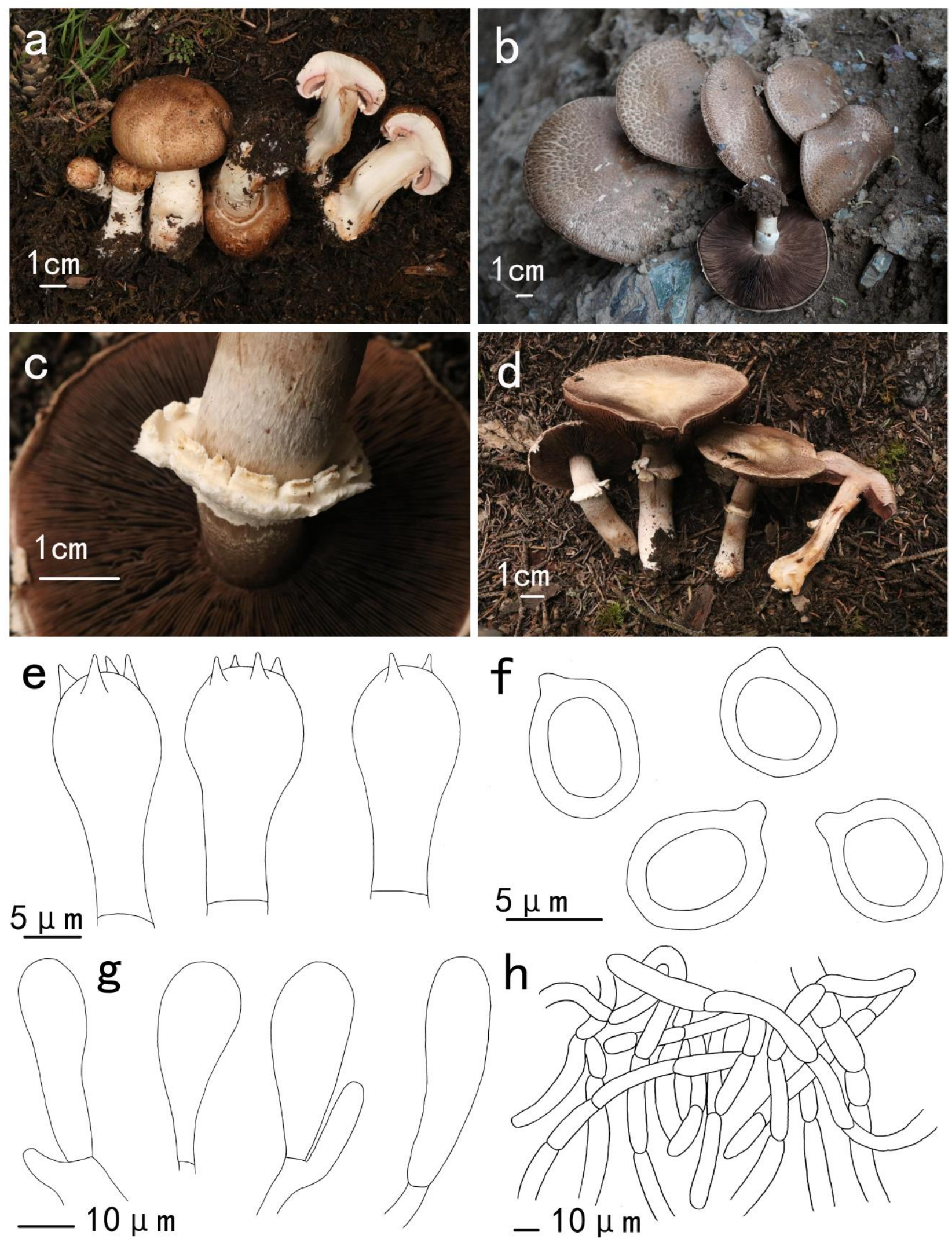

Figure 2 - Morphological characters of Agaricus sinotetrasporus. (b, e-h Holotype ZRL20161020; a specimen ZRL20162192; c, d ZRL20161862). a-d Morphology in field. e Basidia. f Basidiospores. g Cheilocystidia. h Pileipellis hyphae.

Other materials examined - China, Gansu Province, Zhangye City, Sunan county, Qilian Mountain National Natural Reserve, Kangle grassland, Alt. collected by R.L. Zhao, 28 August 2016, 
ZRL20161019 (HMAS 255213), ZRL20161024 (HMAS 255158); ZRL20161797 (HMAS 255157); ZRL20161810 (HMAS 255212). Gansu Province, Zhangye City, Sunan county, Qilian Mountain National Natural Reserve, Yanzhi Forest Park, Alt. 2785 m, E101 ${ }^{\circ} 14^{\prime} 20^{\prime \prime}$, N38 $25^{\prime} 36^{\prime \prime}$, collected by R.L. Zhao, 31 August 2016, ZRL20162094 (HMAS 255214).

Notes - This new species is similar to A. bisporus var. bisporus in morphology, such as characters of cheilocystidia, basidiospores and pileus, especially both have the 2-spored basidia. However, the proposed new species A. qilianensis presents floccose to fibrillose squamose on the stipe surface especially when young, and related long stipe compared with the width of pileus; while those of $A$. bispores is smooth or silky and related shorter stipe (Parra 2013). Furthermore, the phylogenetic analysis indicates they are different species (Fig. 1). A preliminary cloning experiment for its MIP gene PCR amplification products shows the presence of SNP (Single nucleotide polymorphyism). Then we could exclude $A$. qilianensis has a homothallic life cycle.

Agaricus bisporus (J.E. Lange) Imbach, Mitt. naturf. Ges. Luzern 15: 15 (1946)

Fig. 4

Pileus $30-80 \mathrm{~mm}$ in diam., $8-15 \mathrm{~mm}$ thick at the disc, circular, convex to planate, margin decurved, edge exceeding and entire; surface dry, covered by fibrils completely and broken into fibrillose scales, thick at disc and fading toward the margins, brown, ochreous brown. Lamellae free, narrow and crowded, unequal, intercalated with lamellulae, pink, pinkish brown, brown to dark brown. Stipe 40-70 $\times 8-13 \mathrm{~mm}$, cylindrical, hollow, surface smooth or slightly fibrillose. Annulus membranous when young, then thick, intermediate, $5-8 \mathrm{~mm}$ in diam, entire, in median or apical position of stipe, slightly striate at the upper side; Context fresh, firm, white. Discoloration reddish brown on touching and cutting, Odour pleasant, mushroomy.

Macrochemical reactions $-\mathrm{KOH}$ and Schäffer's reactions negative.

Basidiospores 6.6-8.3 × 4.9-6.3 $\mu \mathrm{m}[\mathrm{x}=7.3 \pm 0.6 \times 5.4 \pm 0.4, \mathrm{Q}=1.2-1.5, \mathrm{Qm}=1.4 \pm 0.1, \mathrm{n}$ $=20$ ], ellipsoid, brown, smooth and thick-walled, without germ pore. Basidia 16-22 $\times 5.8-8.0 \mu \mathrm{m}$, clavate, 2-spored. Cheilocystidia $18-36 \times 9.2-13 \mu \mathrm{m}$, mostly clavate, a few pyriform, hyaline. Pleurocystidia absent. Pileipellis a cutis composed of hyphae 7.8-15 $\mu \mathrm{m}$ in diam., long cylindrical, curved, unbranched, containing brown pigments.

Habitat - scattered in Picea crassifolia forest.

Materials examined - China, Gansu Province, Zhangye City, Sunan county, Qilian Mountain National Natural Reserve, Dayekou reserve station, Alt, 2810 2980m, collected by R.L. Zhao, 1 September 2016, ZRL20162049 (HMAS 255152); Gansu Province, Zhangye City, Sunan county, Qilian Mountain National Natural Reserve, Kangle grassland, Alt. collected by R.L. Zhao, 28 August 2016, ZRL20161813 (HMAS 281179), ZRL20161854 (HMAS 279134).

The morphology of those specimens is matching A. bisporus well, and especially match the variety of $A$. bisporus var. bisporus, due to their 2-spored basidia and basidiospores with a length more than $6 \mu \mathrm{m}$ (Callac et al. 2003; Parra 2008). However, in the molecular analysis they cluster with A. bisporus var. burnettii, which is a variety only known from western of the USA and characterized by 4-spored basidia and basidiospores with a length shorter than $6 \mu \mathrm{m}$ (Callac et al. 2003; Parra 2008). In our present study, all those three varieties of A. bisporus are clustered together with full support (Fig. 1). However, the separation of varieties is still not clear in phylogeny. Then in this study we identified those Chinese specimens as A. bisporus, and the definition of variety needs a further study on their lifecycles even if a homothallic life cycle could be excluding, based on preliminary cloning analysis on MIP gene sequences.

Agaricus devoniensis P.D. Orton, Trans. Br. Mycol. Soc. 43(2): 173 (1960)

Fig. 5

Pileus 25-30 $\mathrm{mm}$ in diam., 5-7 $\mathrm{mm}$ thick at the disc, circular, globose, convex, subumbonate, margin inflexed and entire surface dry, silky fibrillose to smooth, white to grey-white in age. Lamellae free, close, unequal, pink, pinkish brown. Stipe 30-35 × 8-10 mm, cylindrical, hollow, surface smooth or slightly fibrillose, white. Annulus membranous, 1-2 $\mathrm{mm}$ in diam, simple, fringed, inferous, superiors, peronate or subperonate, white; Context fresh, firm, white. Discoloration unknown on touching and cutting. Odour pleasant, mushroomy. 

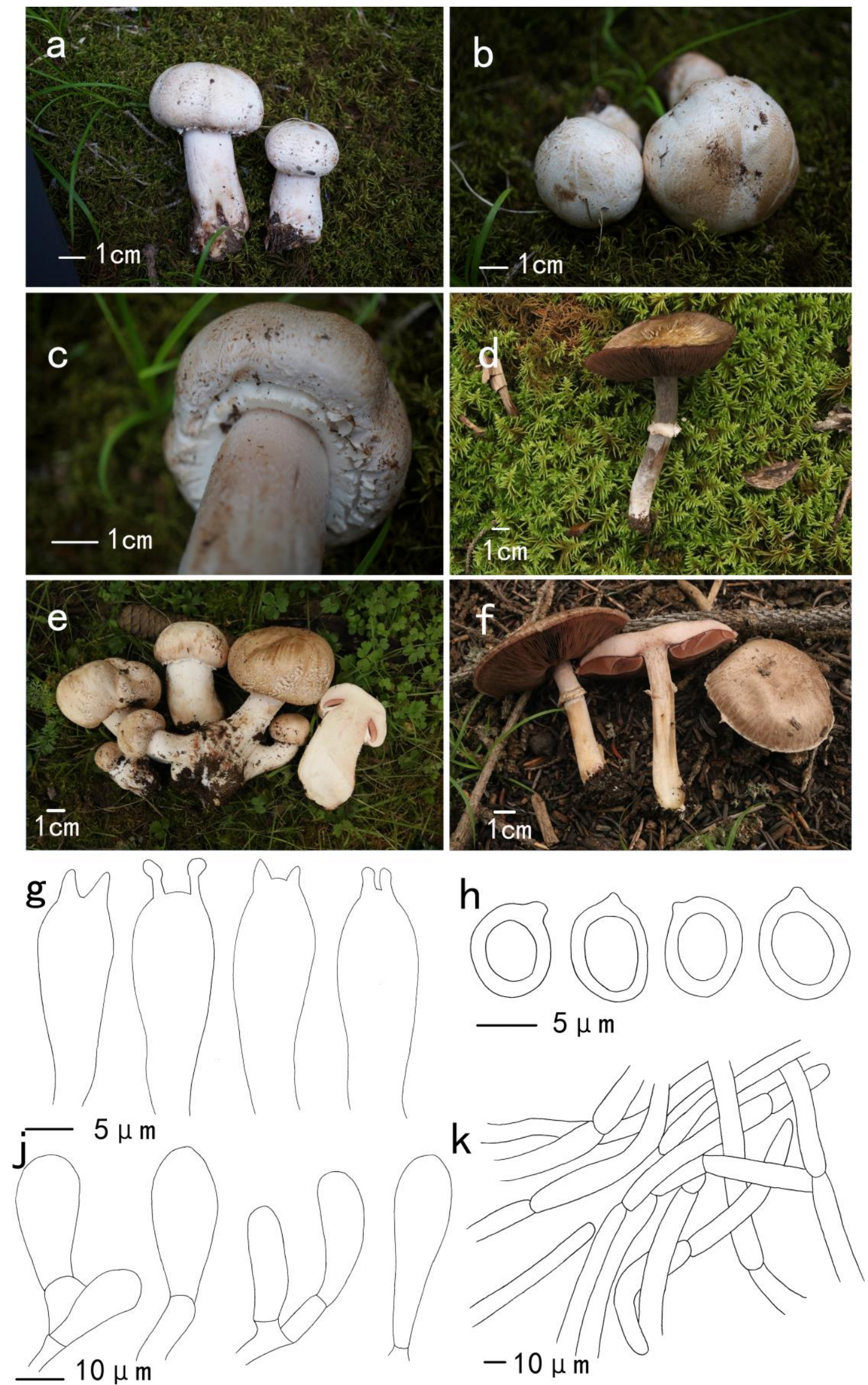

Figure 3 - Morphological characters of A. qilianensis sp. nov. (a-c, g-k Holotype ZRL20161021, d specimen ZRL20161797, e specimen ZRL20161810, f specimen ZRL20162094) a-f Morphology in field. g Basidia. h Basidiospores. j Cheilocystidia. k Pileipellis hyphae. 

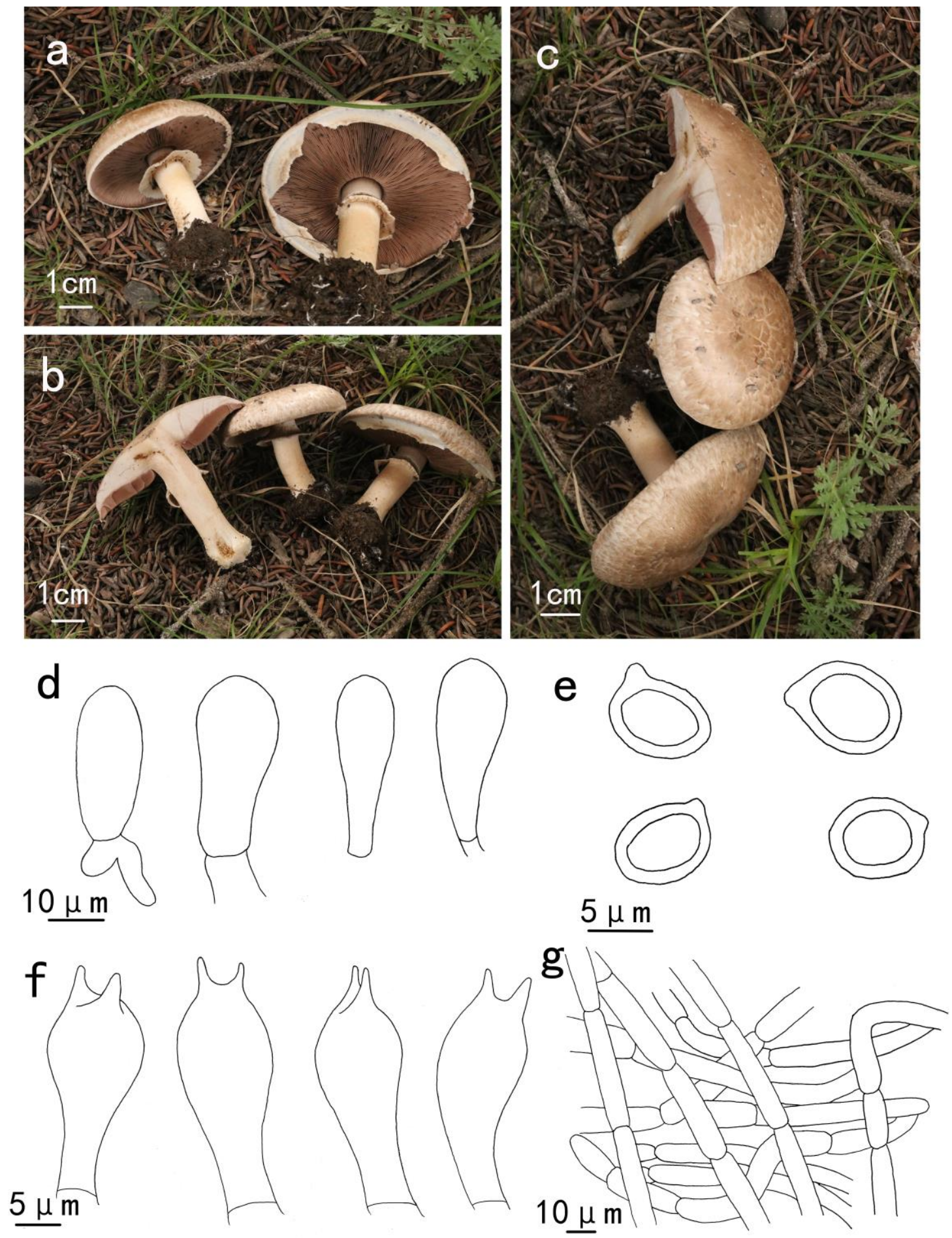

Figure 4 - Morphological characters of A. bisporus (specimen ZRL20162049) a-g Morphology in field. d Cheilocystidia. e Basidiospores. f Basidia. g Pileipellis hyphae.

Macrochemical reactions $-\mathrm{KOH}$ and Schäffer's reactions negative.

Basidiospores 3.9-5.9 × 3.4-5.2 $\mu \mathrm{m}[\mathrm{x}=5.0 \pm 0.4 \times 4.2 \pm 0.4, \mathrm{Q}=1.1-1.3, \mathrm{Qm}=1.2 \pm 0.1, \mathrm{n}$ $=20]$, ellipsoid, brown, smooth and thick-walled without germ pore. Basidia 15-20 × 5.6-7.5 $\mu \mathrm{m}$,

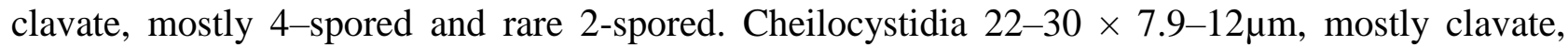
pyriform in some case with long stipe, hyaline. Pleurocystidia absent. Pileipellis a cutis composed of hyphae 8.5-20.5 $\mu \mathrm{m}$ in diam., long cylindrical, curved, unbranched, containing brown pigments.

Habitat - scattered in Picea crassifolia forest. 
Materials examined - China, Gansu Province, Zhangye City, Sunan county, Qilian Mountain National Natural Reserve, Dayekou reserve station, Alt. 2810 2980m, E100 14'31.13", N 38³2'52.71", collected by R.L. Zhao, 31 August 2016, ZRL20162196 (HMAS 279135).

Note - The morphology of Chinese specimen matches the description of A. devoniensis well (Parra 2008) and the molecular analysis also confirms it is A. devoniensis because Chinese specimen cluster with A. devoniensis from Europe under fully support (Fig. 1). This species originally described in Europe is firstly recorded from China.
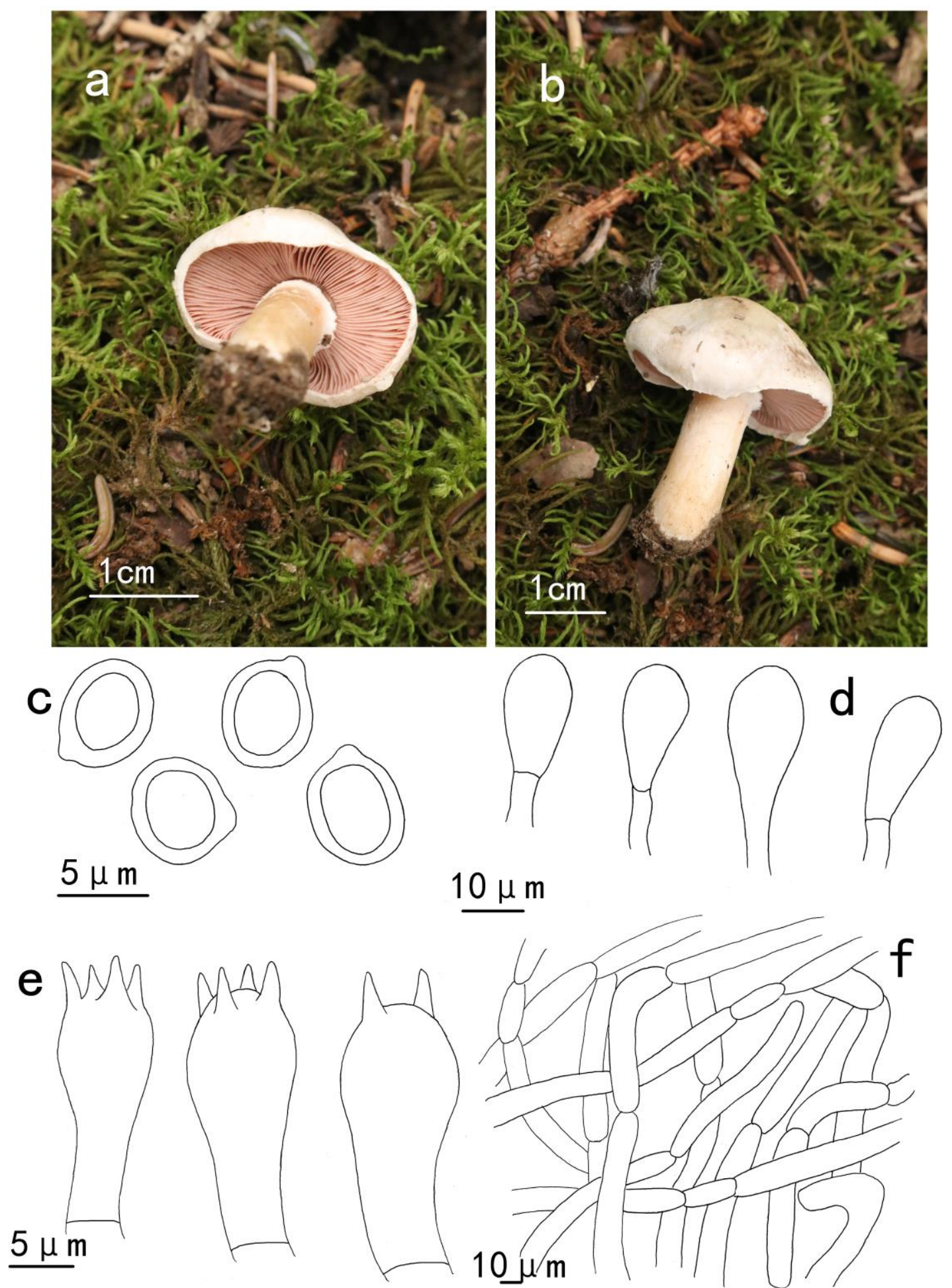

$10 \mu \mathrm{m}$

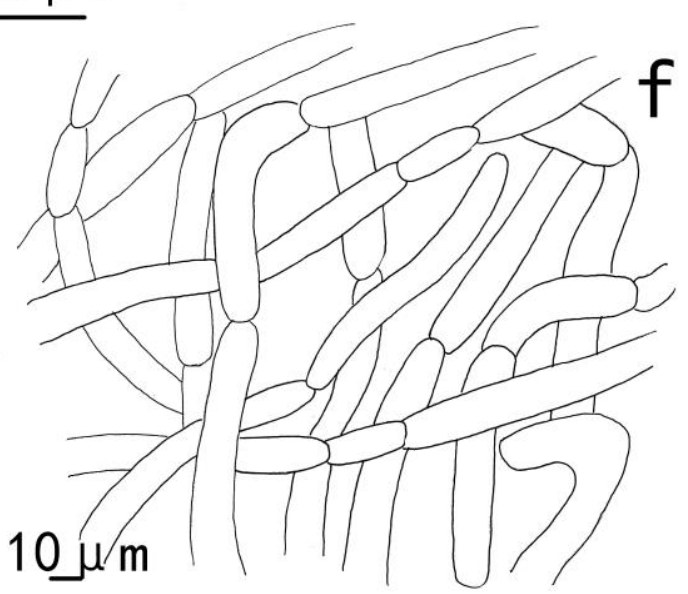

Figure 5 - Morphological characters of A. devoniensis (specimen ZRL20162196) a-f. Morphology in field, c. Basidiospores d. Cheilocystidia, e. Basidia, f. Pileipellis hyphae. 


\section{Discussion}

As memtioned in the above the members of $A$. section Bivelares are edible and some of them are widely cultivated. Species from this section mostly distribute in temperate areas. Similarity, all species reported from this paper are from primary forests of Gansu Province where is in the western China and northern edge of Tibet Plateau, which belong to temperate climate too. In those areas, the vegetational types are simple and almost only composed of Picea species, there are highly species diversity in $A$. section Bivelares. Up to now three new species from this section have been reported from this area, and they are A. sinotetrasporus and A. qilianensis from this study, and $A$. taeniatus in a previous study ( $\mathrm{Li}$ et al. 2014). Some known species which origin from Europe and Northern America also have been found in Qilian Mountain, such as A. devoniensis, A. bisporus. Then their biogeographic information would be interesting to the further study on Agaricus species origin and dispersal.

The previous studies showed the important cultivated species A. bisporus has several varieties and their lifecycles represent a critical character to separate this species into variety level (Callac et al. 2003, Challen et al. 2003). For example, A. bisporus var. bisporus is bisporic amphithallic and mostly pseudohomothallic; while A. bisporus var. eurotetrasporus and A. bisporus var. burnettii are both tetrasporic but the former is homothallic and the latter is amphythallic and mostly heterothallic with smaller spores (Callac et al. 2003). In this study, we could exclude Chinese A. bisporus has a homothallic life cycle. In the future work, their lifecycle should be studied in details, and that would be helpful not only in the identification at the varietal level, but also to develop them as newly cultivated food source.

Supplementary note - In the paper "Characterization of four species including one new species of Agaricus subgenus Spissicaules from Eastern China", published in the volume 7(4) of Mycosphere, the authors failed to indicate the registration number of the species Agaricus catenariocystidiosus which they newly described, so here is indicated a registration number for this name.

Agaricus catenariocystidiosus R.C. Dai \& R.L. Zhao, Mycosphere 7(4): 407

Fig. 2 Names of Fungi: NF570332

\section{Acknowledgements}

This work was supported by grants from the National Natural Science Foundation of China to RLZ (Project IDs 31470152 and 31360014), GJL (Project ID 31500013), The Key Lab Supporting Project from Science and Technology Department of Gansu Province (Project ID 145RTSG005), the Innovative Group of Edible Mushrooms Industry of Beijing (Project ID: BAIC05-2017) and the Opening Foundation of State Key Laboratory of Mycology, Institute of Microbiology Chinese Academy of Sciences. The authors are grateful to Z.L. Ling for assistance in lab work.

\section{References}

Callac P, Jacobé de Haut I, Imbernon M, Guinberteau J et al. 2003 - A novel homothallic variety of Agaricus bisporus comprises rare tetrasporic isolates from Europe. Mycologia 95(2), 222231.

Cappelli A. 1984 - Agaricus L.: Fr. (Psalliota Fr.). Saronno. Libreria editrice Biella Giovanna.

Challen M, Kerrigan RW, Callac P. 2003 - A phylogenetic reconstruction and emendation of Agaricus section Duploannulatae. Mycologia 95(1), 61-73.

Chen J, Callac P, Parra LA. 2017 - Study in Agaricus subgenus Minores and allied clades reveals a new American subgenus and contrasting phylogenetic patterns in Europe and Greater Mekong Subregion. Persoonia 38, 2017, 170 -196.

Didukh M, Wasser SP, Nevo E. 2004 - New data on rare and debatable species of the family Agaricaceae (Fr.) Cohn. Ukrayins'k. Bot. Zhurn. 61(1), 89-99. 
Gardes M, Bruns TD. 1993 - ITS primers with enhanced specifiCity for basidiomycetes application to the identification of mycorrhizae and rusts. Molecular Ecology 2, 113-118.

Gaurav V, David J, Lohman RM. 2010 - SequenceMatrix: concatenation software for the fast assembly of multigene datasets with character set and codon information. Cladistics 27(2), $171-180$.

Heinemann P. 1978 - Essai d'une clé de determination des genres Agaricus et Micropsalliota. Sydowia 30, 6-37.

Nylander JAA. 2004 - MrModeltest v2. Program distributed by the author. Evolutionary Biology Centre, Uppsala University.

Kerrigan RW. 1989 - Studies in Agaricus IV: new species from Colorado. Mycotaxon 34, 119128.

Kerrigan RW, Callac P, Parra LA. 2008 - New and rare taxa in Agaricus section Bivelares (Duploannulati). Mycologia 100(6), 876-892.

Li SF, Xi YL, Qi CX, Liang QQ et al. 2014 - Agaricus taeniatus sp. nov., a new member of Agaricus sect. Biverales from northwest China. Mycotaxon 129(1), 187-196.

Parra LA. 2008 - Agaricus L.: Allopsalliota Nauta \& Bas. Fungi Europaei, vol. 1. Alassio, Edizioni Candusso.

Parra LA. 2013 - Agaricus L.: Allopsalliota Nauta \& Bas, Fungi Europaei, vol. 1A. Alassio, Edizioni Candusso.

Ronquist F, Heulsenbeck JP. 2003 - MrBayes 3: Bayesian phylogenetic inference under mixed models.Bioinformatics 19, 1572-1574. http://dx.doi.org/10.1093/bioinformatics/btg180

Rambaut A. 2016 - Tree Figure Drawing Tool version 1.4.3. Institute of Evolutionary Biology, University of Edinburgh. http://tree.bio.ed.ac.uk/

Silvestro D, Michalak I. 2012 - raxmlGUI: A graphical front-end for RAxML. Organisms Diversity \& Evolution 12, 335-337.

Thongklang N, Hoang E, Estrada AE, Sysouphanthong P et al. 2014 - Evidence for amphithallism and broad geographical hybridization potential among Agaricus subrufescens isolates from Brazil, France and Thailand. Fungal Biology 118(12), 1013-1023.

Wang ZR, Parra LA, Callac P, Zhou JL et al. 2015 - Edible species of Agaricus (Agaricaceae) from Xinjiang Province (Western China). Phytotaxa 202 (3), 185-197.

Wasser SP. 1980 - Flora Fungorum RSS Ucrainicae. Kiev, Naukova Dumka.

White TJ, Bruns T, Lee S, Taylor J. 1990 - Amplification and direct sequencing of fungal ribosomal RNA genes for phylogenetic 315-322 in: MA Innis et al. (eds). PCR protocols: a guide to methods and applications. San Diego, Academic.

Xi YL, Wang ZJ, Yu HP, Wei SL. 2011 - A preliminary report on the study of the large fungus resources in Qilian Mountains National Nature Reserve. Edible Funfi of China 30 (4), 7 13.

Zhao RL, Karunarathna SC, Raspé O, Parra LA et al. 2011 - Major clades in tropical Agaricus. Fungal Diversity 51, 279-296.

Zhao RL, Hyde KD, Karunarathna SC, Desjardin DE et al. 2012 - Agaricus flocculosipes sp. nov., a new potentially cultivatable species from the palaeotropics. Mycoscience 53 (4), 300-311.

Zhao RL, Zhou JL, Chen J, Margaritescu S et al. 2016 -Towards standardizing taxonomic ranks using divergence times - a case study for reconstruction of the Agaricus taxonomic system. Fungal Diversity 78 (1), 239-292. 